Article

\title{
Achieving Complete and Near-Lossless Conversion from IFC to CityGML ${ }^{\dagger}$
}

\author{
Rudi Stouffs $*, \ddagger$ (D) Helga Tauscher $\ddagger$ (D) and Filip Biljecki $\ddagger$ (D) \\ Department of Architecture, School of Design and Environment, National University of Singapore, \\ Singapore 117566, Singapore; helga.tauscher@nus.edu.sg (H.T.); filip@nus.edu.sg (F.B.) \\ * Correspondence: stouffs@nus.edu.sg; Tel.: +65-6601-3513 \\ $+\quad$ Tauscher, H.; Stouffs, R. An IFC-to-CityGML triple graph grammar. In Computing for a better tomorrow: \\ Proceedings of the 36th eCAADe conference; Lodz, Poland, September 2018. \\ $\ddagger \quad$ All authors contributed equally to this work.
}

Received: 31 May 2018; Accepted: 20 August 2018; Published: 27 August 2018

\begin{abstract}
The Singapore Government has embarked on a project to establish a three-dimensional city model and collaborative data platform for Singapore. The research herein contributes to this endeavour by developing a methodology and algorithms to automate the conversion of Building Information Models (BIM), in the Industry Foundation Classes (IFC) data format, into CityGML building models, capturing both geometric and semantic information as available in the BIM models, and including exterior as well as interior structures. We adopt a Triple Graph Grammar (TGG) to formally relate IFC and CityGML, both semantically and geometrically, and to transform a building information model, expressed as an IFC object graph, into a city model expressed as a CityGML object graph. The work pipeline includes extending the CityGML data model with an Application Domain Extension (ADE), which allows capturing information from IFC that is relevant in the geospatial context but at the same time not supported by CityGML in its standard form. In this paper, we elaborate on the triple graph grammar approach and the motivation and roadmap for the development of the ADE. While a fully complete and lossless conversion may never be achieved, this paper suggests that both a TGG and an ADE are natural choices for supporting the conversion between IFC and CityGML.
\end{abstract}

Keywords: building model; BIM; IFC; CityGML; ADE; conversion; triple graph grammar

\section{Introduction}

The Singapore Government has embarked on a project to establish a three-dimensional city model and collaborative data platform for Singapore. The aim of this Virtual Singapore project is to achieve a 3D digital platform that will "enable users from different sectors to develop sophisticated tools and applications for test-bedding concepts and services, planning and decision-making, and research on technologies to solve emerging and complex challenges for Singapore" [1]. Using both airborne and mobile mapping surveys, the current incarnation of the Virtual Singapore model is as a Level of Detail 2 (LoD2) CityGML model. Current endeavors aim to create a semantically enriched LoD3/4 CityGML model. In this paper, we elaborate on a research project to develop a methodology and algorithms to automate the conversion of Building Information Models (BIM) into CityGML building models, capturing both geometric and semantic information as available in the BIM models, including exterior as well as interior structures such as corridors, rooms, internal doors, and stairs.

We target the conversion of BIM models in the Industry Foundation Classes (IFC) data format for openBIM, instead of the native BIM models resulting from specific BIM software applications, e.g., Revit and ArchiCAD. While automation from IFC to CityGML has been demonstrated before 
(e.g., [2-4]), mainly for exterior building shells, this research focuses on achieving a mapping for both exterior and interior structures in LoD3/4, informed by potential use cases and aiming to be as complete and lossless as can be reasonably achieved. Specifically, we are working in close collaboration with a few government agencies in Singapore in order to gain an understanding of their intentions and use cases for the converted BIM models, in particular, and Virtual Singapore, in general. From these use cases, we investigate the development of a CityGML Application Domain Extension (ADE) for building models in order to allow for the representation of semantic information beyond what CityGML currently provides for. Although there have been previous attempts to define such an ADE supporting the conversion and storage of CityGML models obtained from IFC [2,3], these have limitations with respect to the needs and requirements we are identifying. We also aim to demonstrate and prove the conversion routines on existing BIM models obtained from these agencies, specifically, the Housing and Development Board (HDB), Singapore's public housing authority, and the JTC Corporation (JTC), Singapore's lead planner and developer of industrial sites. Both have adopted some quality assurance guidelines with respect to the development of BIM models which, on the one hand, should facilitate the automated conversion process and, on the other hand, provide insights into the limitations of such guidelines with respect to this automation process and the impact of different guidelines and practices on how buildings and their indoor structures are modelled. In principle, we target the conversion of BIM models in the IFC data format, instead of the native BIM models as available from HDB and JTC. Nevertheless, we recognize that there is some advantage in starting from native BIM models and controlling, to some extent, the export of these models to IFC. Specifically, we are investigating BIM requirements and export configurations that result in high-quality IFC4 models from these native BIM models. Finally, we have adopted triple graph grammars as a methodology and technique to flexibly and iteratively develop the rules and algorithms for conversion in order to improve the degree of automation and completeness of the mapping process.

Overall, the project considers three main components: the export from native BIM to IFC, the automated conversion from IFC to CityGML, and the specification of an ADE to enrich the CityGML format in the context of Virtual Singapore (Figure 1). The research and development process takes two parallel approaches (Figure 2). On the one hand, from use cases identified in collaboration with government agencies in Singapore, we identify minimal requirements for the representation of the building model in CityGML and the specification of the ADE. Requirements with respect to the CityGML model are consequently translated into data requirements for the IFC model. These in turn translate into data requirements for the native BIM models. On the other hand, based on the data requirements for the native BIM models, we identify manual steps that need to be taken to ensure a given BIM model meets these requirements. We also determine filtering and export methods that assure a valid IFC model that meets most if not all the data requirements imposed on the IFC model. Next, the triple graph grammar approach is applied to automatically convert the IFC model into a CityGML building model. The result is then demonstrated to our collaborators in order to gain feedback and improve upon the processes where necessary.

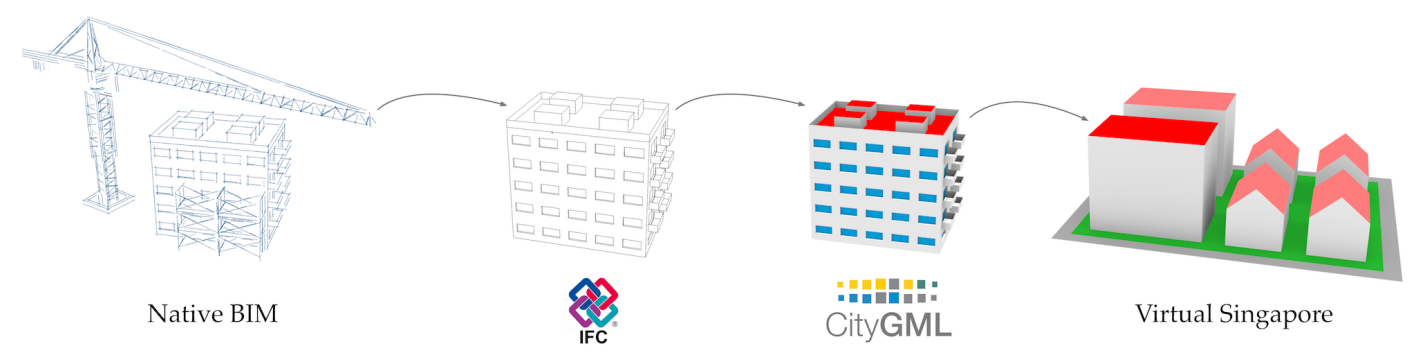

Figure 1. The project in a nutshell: from native BIM (Building Information Model) to the integration of CityGML models in Virtual Singapore. 
In this paper, we focus on the triple graph grammar approach for the conversion from IFC to CityGML and the development of an ADE to extend the CityGML data model (CityGML is a topographic format meaning that the conversion from IFC entails loss of information that might be useful in the geospatial world). In doing so, we reflect on the notion of a complete and near-lossless mapping from BIM to CityGML in light of varying use cases.

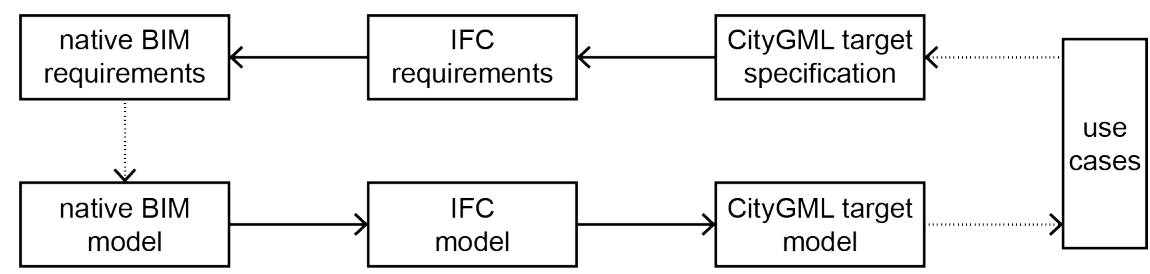

Figure 2. A flowchart of the project: from use cases to native BIM requirements and from a native BIM model to a CityGML model and its uses.

\section{State of the Art: IFC $\rightarrow$ CityGML Conversion}

There is a large number of papers published on the integration of BIM and GIS, a topical subject in the field in the past decade [5]. However, not all researchers deal with the conversion of data from IFC to CityGML (e.g., they employ other formats, integrate both worlds in a unified model [6] or through queries over the shared spatial context [7]). We have identified a set of 17 publications dealing with the conversion from BIM to GIS [2-4,6,8-22].

Out of these 17, the predominant majority (14) employs CityGML. The prevalence of CityGML on the GIS side of the pipeline is not surprising, as the comprehensive review on BIM $\leftrightarrow$ GIS integration by Liu et al. [5] suggests that CityGML is the flagship 3D GIS format when converting from IFC. A few papers are summarised in the continuation of this section.

Donkers et al. [4] developed an open source solution for conversion from IFC to CityGML and demonstrate the automatic generation of valid and semantically rich CityGML LoD3 building models. The conversion is output-driven insofar that it constructs a proper CityGML model using only the information that is needed for a successful conversion, rather than input-driven, considering what IFC information is available for conversion into a CityGML model. Specifically, Donkers et al. [4] elaborate an IFC to CityGML LoD3 transformation in three stages. In the first stage, relevant building elements are extracted using semantic filtering. In the second stage, a volumetric representation of the complete building is generated through geometric transformation using Boolean and morphological operations like dilation and erosion. In the third stage, a refinement process is applied to guarantee the compliance with ISO 19107:2003. The process is focused on LoD3 with an outlook to LoD4.

Deng et al. [3] present a method for automatic data mapping between IFC and CityGML. Mapping rules are established using an instance-based method based on matching comparable components. A notable feature of the work is the generation of CityGML data in multiple LoDs, which is useful for some applications whose performance may be compromised by a high LoD [23]. Furthermore, the researchers develop a CityGML Application Domain Extension (ADE) in order to preserve the semantic information from IFC that cannot be stored in CityGML in a native way.

Several studies $[9,14,24]$ have emphasized that a formal framework for strict semantic and geometric conversion is required for a complete integration of CityGML and IFC-BIM. While it is possible to semi-automate part of the semantic mapping between IFC and CityGML itself, for example, using linguistic and text-mining techniques [25], only a manual method of semantic mapping can guarantee accuracy. We consider a formal framework based on Triple Graph Grammars that can support a variety of use cases through varying rule sets. 


\section{A Triple Graph Grammar Approach}

In this section we first introduce Triple Graph Grammars (TGGs), second illustrate the TGG approach in context of integrated building (IFC) and city (CityGML) models with an example. Third we describe how we operationalise the TGG approach for the conversion from IFC to CityGML. We then show our current progress in developing TGG rule sets, the implementation of a rule-based conversion tool and the application to sample data. Finally we discuss the advantages and limitations of the approach.

\subsection{TGG Introduction, Formal Concepts and Related Work}

Triple graph grammars were first introduced by Andy Schürr in 1995 [26] as a formalism to specify correlations between graph-like data structures and to integrate and transform these potentially disparate structures based on their correlations. Since many software concepts can be expressed as graphs, graphs lend themselves naturally to the application in Computer-aided software engineering (CASE), representing, for instance, entity relationship (ER) models, structure charts, control flow diagrams, or decision trees.

In the context of model-driven engineering focusing on domain models and object-oriented software development, the graphs are to represent meta and instance models. Integration and transformation then pertain to different domain models (here IFC and CityGML), and the basic graph model is to be extended to a typed graph structure in order to represent the type and object graphs, e.g., as proposed by Bardohl et al. [27]. Grammars describe how to form strings of a formal language with production rules, whereas graph grammars adapt this idea for graphs. A graph grammar thus consists of a start graph and a set of production rules which are applied successively to transform the graph. For triple graph grammars, these rules operate on a triad of graphs: two graphs of different type and a third graph that correlates the first two graphs.

The TGG approach has been successfully applied in an engineering context to convert tunnel excavation simulation models from SysML to AnyLogic [28] although the researchers did not follow up the conversion approach after their first experiment [29]. Amelunxen et al. [30] suggest to use graph transformations to check and enforce MATLAB Simulink modelling guidelines as applied in the automotive industry. They consider a modified TGG version (VTGG) to keep the model and its logical views consistent [31]. Aschenbrenner and Geiger [32] developed TGG rules to convert Filmbox (FBX, a proprietary Autodesk format for 3D animation software) files to OpenSceneGraph (OSG).

Ehrig [33] have applied their algebraic approach to graph transformation [34] in the context of model transformations, first using single type and instance graphs that span both the source and target graph, as well as potentially the correlation graph [33]. Later, they have shown that the same principles apply to triple graph grammars because these can be flattened into equivalent single graph transformation systems [35].

The Object Modelling Group (OMG) promotes model-driven architectures (MDA) where software systems are specified as platform-independent models (independent of operating systems, programming languages, etc.). Model transformation plays a crucial role in this approach, mainly for transformation into platform-specific models. After a request for proposals in 2002, which received eight submissions, a language for Query/Views/Transformations (QVT) was adopted in 2005. This motivated the development, research, survey, and comparison of various model transformation approaches including QVT and graph transformations [36]. Many have pointed out that graph transformations and the adopted QVT-core specification are very similar [37] and can be mapped into each other [38]. Schürr and Klar [39] claim that "the fundamental ideas of TGGs have even been adopted by the OMG in their model transformation language standard QVT". 


\subsection{Application of the TGG Approach in Context of the IFC2CityGML Conversion}

In our research, we adopt triple graph grammars to formally relate IFC and CityGML, both semantically and geometrically, and to transform a building information model, expressed as an IFC object graph, into a city model expressed as a CityGML object graph.

Figure 3 shows an example of such a triad of graphs comprising an IFC object graph (left), a CityGML object graph (right), and a correlation graph (center). Note that, strictly speaking, if the correlation graph would be an independent graph, it could not consist of edges only, but would contain additional nodes with bijective relations to the connected IFC- and CityGML nodes. Here, we omit these nodes for clarity. This example triple graph represents just a simple instance of a building project and a corresponding city model with their spatial structure as consisting of two buildings and each building containing one or two storeys. Objects of the IFC and CityGML models constitute graph nodes and are depicted as rectangles, associations or relationships between objects constitute graph edges and are depicted as lines.

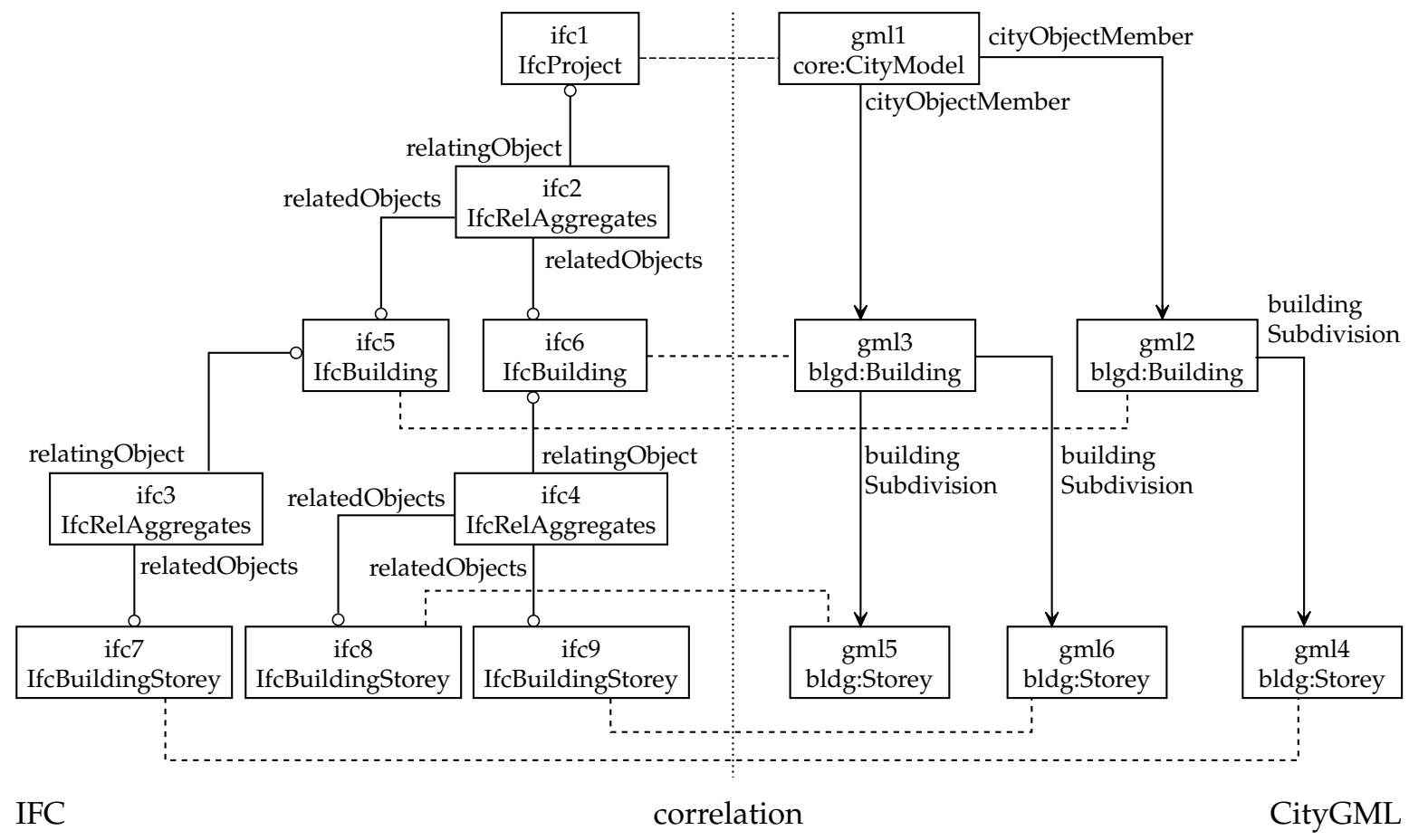

Figure 3. Triple graph consisting of an IFC (Industry Foundation Classes) graph (left, edges with circle end marks), a CityGML graph (right, edges with arrow and marks), and a correlation graph (dashed edges).

In order to distinguish the three graphs, we are employing different edge styles:

- The directed edges of the IFC graph have circle end marks, inspired by Express-G [40]. Note that Express-G is used to document the IFC schema and there is no dedicated graphical representation for instance graphs, thus we are using the schema notation.

- The directed edges of the CityGML graph have arrow end marks, same as associations in UML class and object diagrams [41].

- The undirected edges of the correlation graph are represented as dashed lines without arrow end marks.

The representation further melts the IFC and CityGML instance graphs with the respective type graphs. Each node and edge is annotated with the name of its type. This visual notation and the graph 
model behind typed graph transformation rules has been described in detail for the IFC part [42] and applies similarly to the CityGML graph.

We now introduce a grammar to generate this triple graph. The three rules that comprise this grammar are shown in Figure 4. The initial graph is empty. Rule A creates the root nodes of the hierarchical spatial structures on the IFC and CityGML side and their mutual correlation. Rule B creates a building and Rule $C$ creates a storey in both the IFC and the CityGML graph simultaneously as well as the correlation, including all relevant nodes and edges for all three graphs.

Rule A

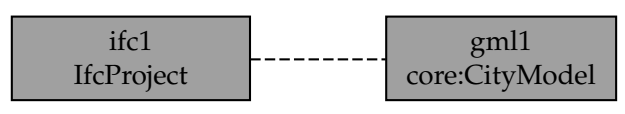

Rule B

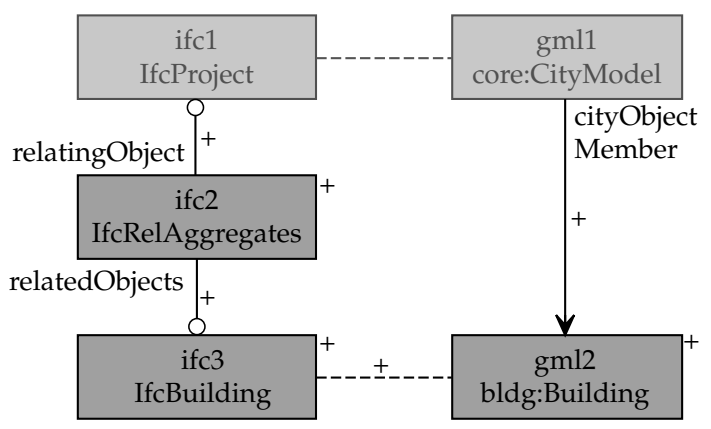

Rule C

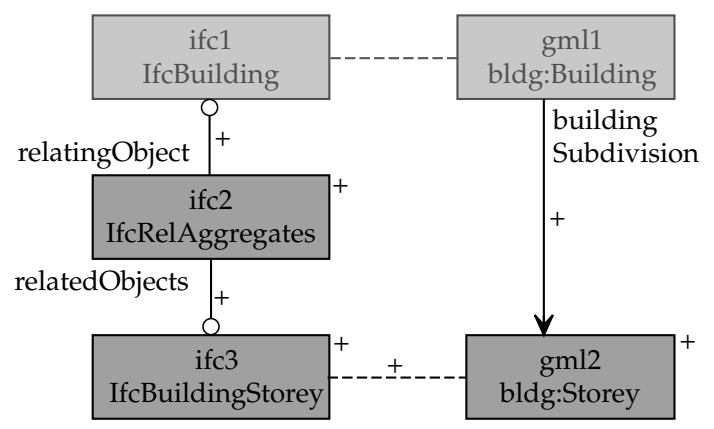

Figure 4. A grammar to create the IFC-CityGML triple graph shown in Figure 3. For each rule, the left-hand-side (indicated in grey) specifies the correlated IFC and CityGML nodes that must exist before rule application; the right-hand-side of the rule (indicated in black with a plus sign) adds correlated IFC and CityGML nodes and their connections into the existing graph triple.

The production rules are depicted as integrated diagrams with the left- and right-hand side of the rule embedded. The left-hand side graph of a rule represents a part of the graph before the application of the rule, namely, what to match during application. The right-hand side represents the same part of the graph after the application of the rule, that is, the replacement. An integrated diagram shows both left- and right-hand side graphs in combination. There is a graphical differentiation between elements that are in both graphs (preserved elements), those only in the left-hand side graph (deleted elements) and those only in the right-hand side graph (created elements). This is a common representation adapted for example in the Graph Transformation Exchange Language GTXL [43]. Some specific productions cannot be accommodated by this representation, but it is sufficient for our purpose. We use grey scales and symbolic annotations to differentiate existing and created nodes and edges in the rule diagram: existing/preserved nodes have middle-grey background and created ones have dark-grey background. "Plus" annotations additionally signify creation. Deleted nodes would receive a light-grey background, with "minus" annotations signifying deletion, but the rule examples in Figure 4 (and below) do not involve the deletion of nodes.

The triple graph from Figure 3 can be derived by first applying rule a to the empty initial graph, then two times rule B with the same match, the initial IfcProject $\leftrightarrow$ CityGML pair. Rule C is then applied three times: two times using the same match of the first building and one time with the second building. By applying the rules in different repetition and order, a potentially infinite number of consistent IFC-CityGML triple graphs similar to the example can be generated. This set is the language described by the grammar. By adding rules we can eventually maximise the part of the IFC and CityGML schema that we cover. 
At this point, there are no denominated source and target graphs. The triple graph rules involves all three graphs, both in the matching, as specified by the left-hand side of a rule, and in the replacement, as specified by the right-hand side. As a consequence, also additions (and deletions) can occur in all three graphs. Typically, triple graph grammars are limited to monotonous or additive productions which do not delete any nodes or edges. Allowing for deletions would add flexibility because intermediate temporary objects would be possible, but it would complicate the formal model.

A triple graph grammar that formally relates two typed object graphs, can be operationalised in different ways [44]:

1. to transform a model of one type into the another,

2. to compute the correspondence between two existing models, or

3. to maintain the consistency between models of the respective types.

We are mainly interested in the transformation Case (1).

In the original TGG paper, Schürr [26] speaks of a forward (LR) transformation, if the source (or input) graph is located on the left side of the graph triple and the target (or output) graph on the right side. Similarly, for a backward (RL) transformation, the source graph is on the right and the target graph on the left. However, both directions are treated equally and there is no precedence involved. To avoid confusion with the left- and right-hand side of a production or transformation rule, we will use the terms source and target or the names of the concrete model types we are dealing with-IFC and CityGML-instead.

For the transformation case, the TGG production rules have to be converted into operational transformation rules. A given IFC graph with empty correlation and CityGML graphs constitutes the start triple. This single graph is then extended into a full consistent graph triple by applying the operational rules and thus populating the CityGML and correlation graph. Schürr [26] has shown that such one-way transformation rules can be derived from the triple graph grammar rules by splitting them into (1) a source-local part that does not involve the target and correlation graph and (2) a source-to-target part that leaves the source graph unchanged. Figure 5 shows an example of such a split: Rule B from the example IFC $\leftrightarrow$ CityGML grammar in Figure 4 is divided into two rules: an IFC-local rule B' which is restricted to the IFC graph and an IFC $\rightarrow$ CityGML transformation rule B" which does not change the IFC graph. Applying the two rules B' and B" sequentially yields the same result as applying the original rule $B$.

Rule B'

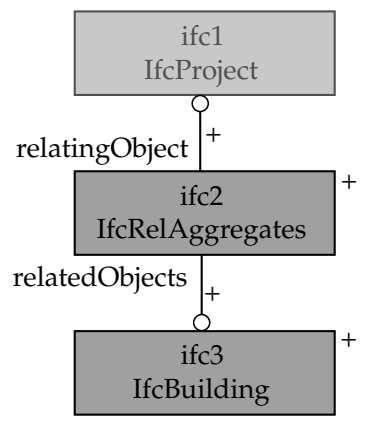

Rule B"

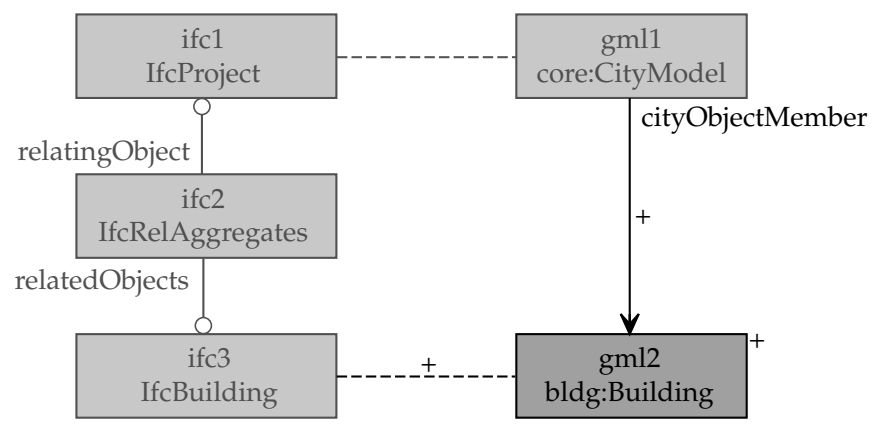

Figure 5. IFC-local rule B' (left) and IFC $\rightarrow$ CityGML transformation rule B" (right) derived by splitting triple graph grammar rule B from Figure 4 .

Informally speaking, this split factors out exactly those parts from the simultaneous triple graph generating rules that build the IFC graph. These parts of the rules are not necessarily needed for the transformation because we already start with a given IFC graph. However, these IFC-local rules constitute a grammar to build all potential source graphs. This grammar can be used to parse the start graph and thus determine the application order of the associated transformation rules. 
The two sides of a rule (left- and right-hand) must be carefully distinguished from the two sides of the triple graph (source and target) since the left- and right-hand side of a rule do not directly correspond to the source and target graph. As can be seen from Figure 5, even for a forward transformation where we conceive of the IFC graph as the source and the CityGML graph as the target, the left-hand-side of the transformation rule, consisting of deleted and preserved graph elements, may include nodes and/or edges from all three graphs. The creation of nodes and edges is however limited to the target graph and the correlation graph, by definition of the splitting operation.

\subsection{Direct Specification of Parsing and Transformation Rules for IFC $\rightarrow$ CityGML Conversion}

Currently, we are only interested in a one-directional IFC $\rightarrow$ CityGML transformation system, with IFC as the source and CityGML as the target metamodel. Therefore, for the implementation of the actual transformation we specify the forward transformation rules directly instead of deducing them from triple graph rules which create all three graphs simultaneously. This short-cut method sacrifices the possibility of flexible bi-directional transformation and synchronization in favour of a straight-forward implementation for just a uni-directional transformation. We presume that the rules can be easily converted to general triple graph grammar rules and thus we could generalize the rule sets for bi-directional transformation and synchronisation.

Recall the example in Figure 5 with the IFC-local rule B' and the IFC $\rightarrow$ CityGML rule B". We now directly specify the IFC $\rightarrow$ CityGML role and use this to guide the traversal of the IFC graph, the generation of the corresponding CityGML nodes and edges, as well as their embedding within the CityGML object graph created earlier in the transformation process.

To enable this, we pose further constraints on the transformation rules. Each transformation rule consists of three sets of nodes and four sets of edges: the existing IFC nodes $N_{I F C}$, the existing GML nodes $N_{G M L}$, the created GML nodes $N_{G M L}^{+}$, the existing IFC edges $E_{I F C}$, the existing correlation graph edges $E_{C O N}$, the created correlation graph edges $E_{C O N}^{+}$, the existing GML edges $E_{G M L}$ and the created GML edges $E_{G M L}^{+}$. The following constraints apply:

1. There are only two correlation graph edges, one in the set $E_{\mathrm{CON}}$ and one in the set $E_{\mathrm{CON}}^{+}$.

2. The edge $e \in E_{C O N}$ connects nodes $n_{1} \in E_{I F C}$ and $n_{2} \in E_{G M L}$.

3. The edge $e \in E_{C O N}^{+}$connects nodes $n_{3} \in E_{I F C}$ and $n_{4} \in E_{G M L}^{+}$.

4. a path exists (taking edges as undirected) from $n_{1}$ to $n_{3}$ via edges in $E_{I F C}$ and nodes in $N_{I F C}$.

5. a path exists (taking edges as undirected) from $n_{2}$ to $n_{4}$ via edges in $E_{G M L}^{+}$and nodes in $N_{G M L}^{+}$.

The example from Figure 5 fulfils these requirements with $n_{1}=i f c 1, n_{2}=g m l 1, n_{3}=$ ifc3, and $n_{4}=g m l 2$.

Another conforming example is shown in Figure 6. This rule identifies an interior wall surface within the IFC graph and creates and embeds a corresponding node in the CityGML graph: an interior wall surface within IFC is first identified as an internal, physical space boundary that relates to a wall and a space and has a connection surface geometry. It is the latter that is then transformed into an interior wall surface node in the CityGML graph; both are related through a correlation edge. Finally, the created wall surface node is connected to the existing room in the GML graph which corresponds to the related space in the IFC graph identified in the beginning.

Given that these requirements are fulfilled for a transformation rule, we do not need to explicitly specify a corresponding IFC-local rule for parsing. Instead of the graph parsing, we can apply the transformation rules directly in a predefined application order as follows. We use the pair $\left(n_{1}, n_{2}\right)$ as an entry point, follow the path to $n_{3}$ in the IFC graph and identify all possible matches of the IFC part of the left-hand side of the rule. For each match we create a corresponding node $n_{4}$ and attach it to $n_{2}$ by creating the required edges and intermediate GML nodes as specified by the rule's right-hand side. Depending on the number of matches, this application generates zero or more pairs of an existing IFC node and a newly created and correlated GML node. These pairs form the entry points for the application of subsequent rules. 
This simplifies the specification of the rules and the implementation process of a first generic transformation algorithm. We might relax these constraints and adjust the transformation algorithm if the need arises in the process developing the specific IFC $\rightarrow$ CityGML transformation rules. Though this approach obviously limits the applicability of these rules to a recursive, hierarchical development, it allows for a straightforward generation of conversion routines from this mapping, and it can easily be extended to include other rule types and rule application strategies.

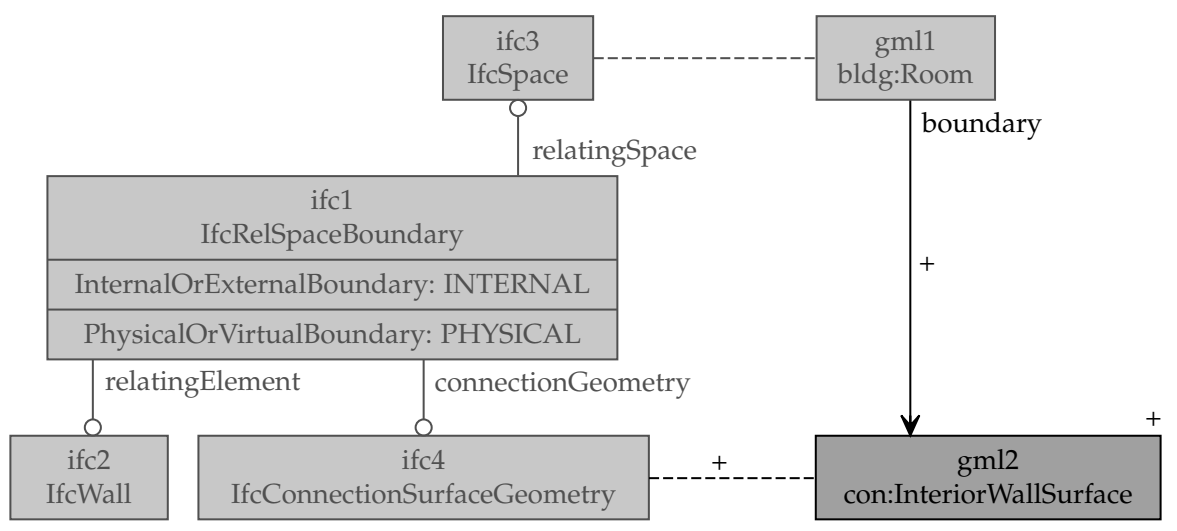

Figure 6. Example of a triple graph rule transforming an interior wall surface from IFC into CityGML. The left-hand-side of the rule (indicated in grey) specifies four IFC nodes, their mutual edges, and a correlated CityGML node; the right-hand-side of the rule (indicated in black with a plus sign) adds a CityGML node and a correlation edge between an existing IFC node and the new CityGML node.

\subsection{Implementation of Rule-Based Conversion, Rule Set Specification and Application to Sample Data}

In a first prototype the conversion was implemented in an imperative way, but already broken into rule-like transformation pieces that can be reused and recombined. In ongoing work we are now changing the implementation to consume graph transformation rules specified in a declarative style.

In parallel we are developing concrete rule sets using a custom domain-specific language (DSL) and rule management platform and repository. A first rule set transforms IFC building elements with their solid geometry straight into semantically classified CityGML surfaces. Other rule sets employ the boundary surfaces in IFC to populate semantic surfaces in CityGML or extract LOD0 floor plans from IFC [45]. Most rule sets replicate the building structure of storeys in CityGML to embed the converted features. Initially the rule sets cover only walls, slabs, and rooms. We intend to successively add more details such as openings.

Before we conduct case studies with real-world data, we test and verify the conversion process on controlled input data. For this, we use (a) the IFC export of the advanced Revit tutorial model, an office building and (b) a handcrafted IFC file of a two-storey residential building that we continuously update according to our progress in rule development. Figure 7 shows conversion results for both data sets, including spaces as well as walls, slabs and roofs.
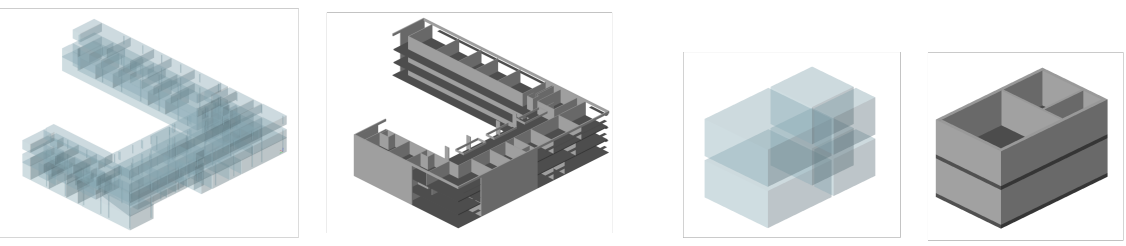

Figure 7. Example conversion results: Revit advanced tutorial office building (left) and handcrafted two-storey residential building (right). Conversion includes spaces, walls, slabs and roofs, but roofs are omitted for illustrative reasons. 


\subsection{Advantages and Limitations of the Approach}

First of all, graph representations provide a solid foundation for formal investigations regarding correctness and completeness of a transformation system. The use of a triple graph grammar allows us to develop the formal mapping from IFC to CityGML incrementally. We can develop different configurations (as rule sets) for different IFC versions or IFC profiles, e.g., specific to a particular MVD or exporting native BIM software, for different CityGML versions, ADEs, and use cases.

From a given set of transformation rules we can extract the IFC-local parts and use these as requirements for IFC source graphs. This allows preliminary checks of whether a given IFC input will yield a result in the conversion to CityGML or it lacks essential content with regard to this specific set of rules. Thus we gain insight in the extent to which an IFC file conforms to the rule set. We could also deduce the requirements as MVDs from the IFC subgraph templates.

Oppositely, we can also check to what extent a rule set is able to cover a given IFC file and thus identify gaps in the rule set. To this end we mark all nodes and edges in the IFC source graph that are matched by any transformation rule. Non-matched nodes and edges indicate blind spots in the rule set with regard to a complete conversion. We can also generate metrics such as percentage of covered nodes and edges.

By converting the operational rules to general triple graph grammar rules the rule sets could be generalised and used for bi-directional transformation and synchronisation of IFC and CityGML graphs. Another advantage of the triple graph grammar approach is that while the rules can be expressed graphically, these can be automatically translated into software routines to perform the actual transformation or used as input from a generic algorithm. At the same time the method constitutes an additional abstraction layer which complicates implementation.

The current transformation rule application mechanism is limited to a hierarchical development. This seems however appropriate for the conversion into an XML-based, inherently hierarchical data model. Further, this limitation might even turn out to be less restrictive, since the correspondence graph is required to be bijective and we include IFC inverse attributes. This way the traversal of the IFC graph can happen freely, not necessarily following the direction of the IFC edges. Rules may not necessarily create new CityGML nodes to preserve correspondence bijectivity and instead just create new edges for existing CityGML nodes, thus digressing from the tree structure. More detailed investigations are necessary.

We also realized that the current approach does not easily accommodate recursive rule applications, such as aggregations with multiple levels.

\section{Development of an ADE}

\subsection{Overview and Related Work}

As it is clear from the introduction of this paper, CityGML and IFC are inherently substantially different formats that are designed for different uses. There is a large disparity between them in terms of object types, scale, resolution, geometry, attributes, and relationships. It then comes with no surprise that the CityGML data model does not support storing all information from its architectural counterpart [12], preventing the preservation of potentially valuable information during the conversion.

Therefore, it would be beneficial to take advantage of the Application Domain Extension (ADE), a native mechanism of CityGML to extend its data model supporting specific purposes, such as enhancing its usability for particular applications such as energy estimations, transportation simulations, and harmonisation of national geographic data information models [46,47].

The principal functionality of the CityGML ADE concept is defining new feature classes and attributes. An example is the definition of the attribute on the cooling system of a building, a piece of information that is not possible to store in CityGML by default, potentially being useful in energy modelling applications [48]. Given the fact that the application of 3D city models is growing 
and encompasses a variety of application domains and software packages [49,50], ADEs are becoming more relevant in supplementing information to CityGML. For more technical details on the ADE concept, the reader is referred to publications $[46,47,51]$.

Therefore, employing the ADE concept in the frame of our project would allow capturing information from IFC that might be relevant in the geospatial context, but at the same time is not supported by CityGML in its standard form. However, a surprisingly small number of the projects cited in Section 2 appear to engage ADEs. Out of the cited 14 papers on IFC $\rightarrow$ CityGML conversion, only four include the development of the ADE mechanism $[2,3,13,18]$, each with its own particularities. For example, de Laat and van Berlo [2] identify a subset of 10\% features in IFC deeming them relevant for the geospatial stakeholders, while Deng et al. [3] give special attention to the structural properties found in IFC and to the LoD of the output CityGML models.

The low rate of use of ADEs in this theatre can be explained by the fact that most related research efforts are not concerned with achieving a complete and near-lossless transformation of information from IFC to CityGML. They merely strive to preserve the set of features that are essential in the CityGML context, and to run geospatial analyses in which extra information provided by IFC is redundant and may even be considered detrimental. The latter observation is deduced from the work of Deng et al., who establish a method for the conversion of IFC to CityGML, resulting in datasets in a LoD lower than LoD4 [3,23].

Even though there is only a handful of ADEs developed to foster the conversion of IFC to CityGML, they provide some potentially useful observations. For example, de Laat and van Berlo [2] point out that only a fraction of IFC features is relevant for the geospatial context, and the authors give a list of those they deem matching that criteria. Such information may be useful in developing an own ADE, since one of the first steps would be the identification of the features that ought to be preserved in the conversion.

\subsection{Arguments for an ADE and Shortcomings}

While the motivation to employ ADE in this project is obvious from Section 4.1, this section continues arguing why an ADE is necessary. Specifically, some of the benefits of ADE in the context of the BIM $\leftrightarrow$ GIS interoperability are that:

1. It is the preferred method of extending the CityGML data model (the other one being the Generics mechanism, which allows generic attributes and objects, but is not without shortcomings [46]);

2. Thanks to the flexible extension of the data model, ADE allows for a (in practice) nearly lossless and strict conversion from IFC to CityGML;

3. ADE supports overcoming the lower structural level than IFC, enabling introduction of new concepts not available in CityGML; and

4. ADE enables storing only the data we are interested in for particular use cases and stakeholders (i.e., it allows being "selectively strict and lossless").

For these reasons, it appears that it is beneficial to work in the direction of employing an ADE to support the conversion. Therefore, it is no surprise that the ADE mechanism has already been used for this purpose (Section 4.1). However, we aim for developing a new ADE to tailor it for the project.

Following the description of advantages, it should also be noted that ADE does not solve all challenges. As argued by different research groups [2,18], the introduction of an ADE only partially mitigates different issues encountered during the conversion, because CityGML cannot capture all the information from IFC. Hence a fully strict and lossless conversion may never be achieved even with a intricate ADE. However, CityGML was never intended to substitute IFC, and in the geospatial context only a subset of IFC is relevant. Therefore, this limitation should not in practice affect much use cases and other aspects. 
Other disadvantages of developing an ADE for this purpose are the ones generic that apply to all ADEs such as the fact that the development of an own ADE entails working on software support as well, and an ADE adds an additional layer of complexity to the CityGML data model [46].

\subsection{Towards an ADE for IFC within This Project}

There are two general approaches of developing an ADE:

- Doing so from scratch developing a "brand new" ADE.

- Modifying and/or extending an existing ADE (see for example the work of Kumar et al. [52] demonstrating that it is possible to customise an existing ADE to match particularities of a national context). Since ADEs are released as UML/XSD they can be further customised/extended and improved by others.

Even though there is existing related work about developing an ADE for fostering interoperability with IFC (Section 4.1), as mentioned in the previous section, it might be beneficial to develop an own ADE for overcoming the aforementioned drawbacks, primarily:

- Obsolescence: related work (such as the GeoBIM extension as one of the most prominent works in this field was published in 2011 [2]) might not be up-to-date anymore.

- Conditioning for CityGML 3.0: while the new version of the standard will probably not affect the ADE concept found in CityGML 2.0 [46], there may be changes that should be taken into account, such as the new LoD concept [53].

- Adapting to the Singapore context: development of a new ADE is not only beneficial to suit various geographical and architectural properties, but also to match properties of input IFC models that are specific to Singapore Government agencies.

- Fitting use cases: adaptation for use cases of interest to the project stakeholders. For example, the use case on planning vegetating roof surfaces (i.e., green roofs) would require information on the current status of the roofs.

In general, the following steps are foreseen when developing an ADE for the purpose of the project:

1. Identification of the relevant features and concepts to be translated from IFC to suit the geospatial context (see [2] for inspiration).

2. Harmonisation with the local context (e.g., taking into account the local architecture and other aspects particular to a given geographic setting).

3. Harmonisation with the national standardisation efforts (e.g., include attributes found in official standards).

4. Identification of features relevant for a particular use case and stakeholder.

In the context of our current project discussions, two possibilities and approaches are identified:

- Developing a single all-in ADE that is generic and applicable to a variety of use cases (similar to [2]).

- Developing custom (tailored) ADEs for each stakeholder that would focus on only one stakeholder and one use case at a time, e.g., one that suits the energy use case. This is not an entirely new idea, as some researchers develop such "mini-ADEs" or "light-ADEs" [54,55], which are specialised focusing on a narrow subject.

A simplified excerpt of the preliminary version of the ongoing work on the ADE is given in Figure 8. The ADE extends the CityGML notion of a building with additional properties, such as the information on the parking facilities associated to a building. Some of these properties are specific to Singapore, for example the typology of buildings. 


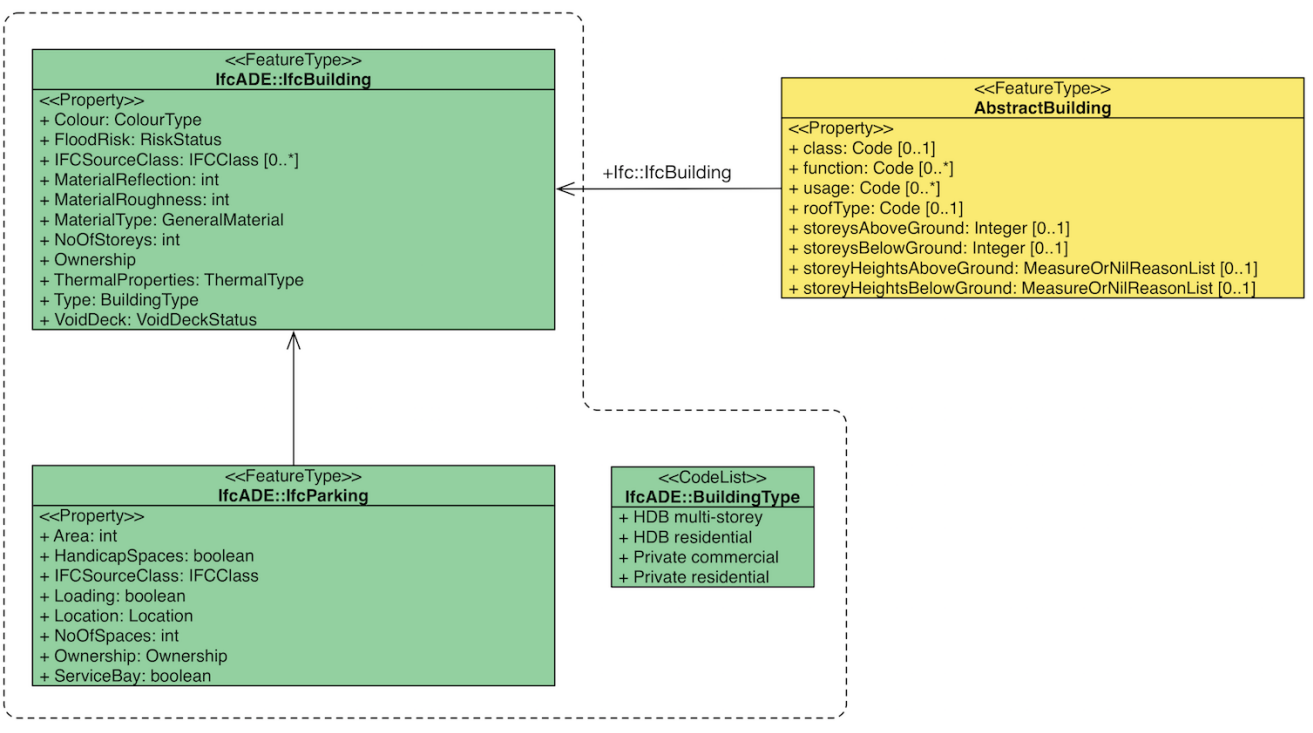

Figure 8. Excerpt from a simple ADE extending the CityGML data model and supporting the conservation of potentially useful information from architectural models and other sources. Such an enrichment of the data model may benefit the usability of the data in certain applications, e.g., pertaining to the local geographical context.

\section{Discussion and Conclusions}

As can be gathered from previous work on IFC $\rightarrow$ CityGML conversion, the difficulty is not so much in the automation, but in achieving a complete and near-lossless mapping between an IFC building model and the corresponding CityGML model (including interior structures). This is especially so in the light of variations in BIM models as developed with different BIM software applications and by different users and practices, and in the light of future requirements on a semantically enriched 3D city (CityGML) model, for example to export information to other formats and applications. Our approach is threefold.

Firstly, we are holding discovery sessions with collaborators and stakeholders in order to identify relevant use cases that provide insight into which information should be included in the mapping and, thus, when the CityGML model may be considered "complete". For example, while a complete breakdown of the material composition of a physical building element is beyond any requirement, various properties that reflect on this material composition or its finishing layer, such as its thermal value, transparency, reflectiveness or roughness value, may be required for specific use cases.

Secondly, we will develop one or more ADEs in order to support the capturing of such information into CityGML, where its standard form does not support this. Specifically, this paper suggests that ADE is a natural choice for supporting the conversion between IFC and CityGML. In fact, ADEs have been previously developed for that purpose. In spite of existing solutions, developing an own ADE tailored for this project is viable and recommended, as discussed in Section 4.2. While all the information from IFC may never be fully stored in CityGML [56], ADE provides a good approach to diminish the loss of information that the conversion to CityGML entails, and supporting applications of stakeholders.

Thirdly, we adopt triple graph grammars as part of a formal framework for strict semantic and geometric conversion. Specifically, we develop a triple graph grammar to formally relate and transform a building information model, expressed as an IFC object graph, into a city model, expressed as a CityGML object graph, and to transform a model of one type into another. Rather than specifying triple graph rules creating all three graphs, the IFC object graph, the CityGML object graph and the correlation graph, simultaneously, instead we specify the forward transformation rules directly and extract the IFC-local rule parts and use these as requirements for IFC source graphs. This allows to conduct preliminary checks of whether a given IFC input will yield a result in the conversion to 
CityGML but also to what extent a rule set is able to cover a given IFC file and thus how complete the conversion is. An additional advantage of the triple graph grammar approach is that it allows an incremental approach to the development of the formal mapping.

The combination of these three approaches provides us with the means to define and achieve, in tandem, a complete and near-lossless conversion from IFC to CityGML. However, the definition of complete and near-lossless remains dependent on the specific use case considered. For this reason, we consider the development of a variety of rule sets, possibly selected and extracted from a single all-encompassing rule repository, to address different use cases. However, we have not determined yet whether varying rule sets for varying use cases would be aided by considering multiple smaller, complementary ADEs instead of a single ADE that addresses all use cases considered. Further research and development is required on the TGG rule set and the ADE, with respect to both different BIM models and practices, and different use cases, to further detail the selected conversion approach and demonstrate its applicability in practice.

Author Contributions: Supervision, R.S.; Writing—original draft, R.S., H.T. and F.B.; Writing—review \& editing, R.S., H.T. and F.B.

Funding: This material is based on research/work supported by the National Research Foundation under Virtual Singapore Award No. NRF2015VSG-AA3DCM001-008.

Acknowledgments: The authors would like to acknowledge the remaining members of the research team: Patrick Janssen, James Crawford, Salman Khalili Araghi, Amol Konde and Kamel Adouane. We gratefully acknowledge the ongoing work of Diana Moraru (Ordnance Survey Great Britain) on the ADE.

Conflicts of Interest: The authors declare no conflict of interest.

\section{References}

1. Virtual Singapore. Available online: https://www.nrf.gov.sg/programmes/virtual-singapore (accessed on 26 March 2018).

2. De Laat, R.; van Berlo, L. Integration of BIM and GIS: The Development of the CityGML GeoBIM Extension. In Advances in 3D Geo-Information Sciences; Springer: Berlin/Heidelberg, Germany, 2011; pp. 211-225.

3. Deng, Y.; Cheng, J.C.P.; Anumba, C. Mapping between BIM and 3D GIS in different levels of detail using schema mediation and instance comparison. Autom. Constr. 2016, 67, 1-21. [CrossRef]

4. Donkers, S.; Ledoux, H.; Zhao, J.; Stoter, J. Automatic conversion of IFC datasets to geometrically and semantically correct CityGML LOD3 buildings. Trans. GIS 2016, 20, 547-569. [CrossRef]

5. Liu, X.; Wang, X.; Wright, G.; Cheng, J.; Li, X.; Liu, R. A State-of-the-Art Review on the Integration of Building Information Modeling (BIM) and Geographic Information System (GIS). ISPRS Int. J. Geo-Inf. 2017, 6, 53. [CrossRef]

6. El-Mekawy, M.; Östman, A.; Hijazi, I. A Unified Building Model for 3D Urban GIS. ISPRS Int. J. Geo-Inf. 2012, 1, 120-145. [CrossRef]

7. Daum, S.; Borrmann, A.; Kolbe, T. A Spatio-Semantic Query Language for the Integrated Analysis of City Models and Building Information Models. In Advances in 3D Geoinformation; Abdul-Rahman, A., Ed.; Springer International Publishing: Cham, Switzerland, 2017; pp. 79-93. [CrossRef]

8. Arroyo Ohori, G.A.K.; Diakité, A.A.; Ledoux, H.; Stoter, J.; Krijnen, T. GeoBIM Project_Final Report; Technical Report; Delft University of Technology: Delft, The Netherlands, 2017.

9. Benner, J.; Geiger, A.; Leinemann, K. Flexible generation of semantic 3D building models. In Proceedings of the 1st ISPRS/EuroSDR/DGPF International Workshop on Next Generation 3D City Models, Bonn, Germany, 21-22 June 2005; Gröger, G., Kolbe, T.H., Eds.; EuroSDR: Leuven, Belgium; pp. 17-22.

10. Ellul, C.; Boyes, G.; Thomson, C.; Backes, D. Towards Integrating BIM and GIS-An End-to-End Example from Point Cloud to Analysis. In Advances in 3D Geoinformation; Springer: Cham, Switzerland, 2017; pp. 495-512.

11. Geiger, A.; Benner, J.; Haefele, K.H. Generalization of 3D IFC Building Models. In 3D Geoinformation Science; Springer: Dubai, UAE, 2015; pp. 19-35.

12. Kang, T.W.; Hong, C.H. A study on software architecture for effective BIM/GIS-based facility management data integration. Autom. Constr. 2015, 54, 25-38. [CrossRef] 
13. Hijazi, I.; Ehlers, M.; Zlatanova, S.; Becker, T.; van Berlo, L. Initial Investigations for Modeling Interior Utilities Within 3D Geo Context: Transforming IFC-Interior Utility to CityGML/UtilityNetworkADE. In Advances in 3D Geo-Information Sciences; Springer: Berlin/Heidelberg, Germany, 2011; pp. 95-113.

14. Isikdag, U.; Zlatanova, S. Towards Defining a Framework for Automatic Generation of Buildings in CityGML Using Building Information Models. In 3D Geo-Information Sciences; Springer: Berlin/Heidelberg, Germany, 2009; pp. 79-96.

15. Kang, T.W.; Hong, C.H. IFC-CityGML LOD mapping automation using multiprocessing-based screen-buffer scanning including mapping rule. KSCE J. Civ. Eng. 2017, 4, 1-11. [CrossRef]

16. Jusuf, S.; Mousseau, B.; Godfroid, G.; Soh, J. Path to an Integrated Modelling between IFC and CityGML for Neighborhood Scale Modelling. Urban Sci. 2017, 1, 25. [CrossRef]

17. Rafiee, A.; Dias, E.; Fruijtier, S.; Scholten, H. From BIM to Geo-analysis: View Coverage and Shadow Analysis by BIM/GIS Integration. Procedia Environ. Sci. 2014, 22, 397-402. [CrossRef]

18. Sebastian, R.; Böhms, M.; van den Helm, P. BIM and GIS for low-disturbance construction. In Proceedings of the 13th International Conference on Construction Applications of Virtual Reality, London, UK, 30-31 October 2013; pp. 469-479.

19. Teo, T.A.; Yu, S.C. The extraction of indoor building information from BIM to OGC IndoorGML. Int. Arch. Photogramm. Remote Sens. Spat. Inf. Sci. 2017, XLII-4/W2, 167-170. [CrossRef]

20. Xu, X.; Ding, L.; Luo, H.; Ma, L. From building information modeling to city information modeling. J. Inf. Technol. Constr. ITcon 2014, 19, 292-307.

21. Yu, S.C.; Teo, T.A. The generalization of BIM/IFC model for multi-scale 3D GIS/CityGML models. In Proceedings of the 35th Asian Conference on Remote Sensing, Nay Pyi Taw, Myanmar, 27-31 October 2014.

22. Wu, B.; Zhang, S. Integration of GIS and BIM for indoor geovisual analytics. Int. Arch. Photogramm. Remote Sens. Spat. Inf. Sci. 2016, XLI-B2, 455-458. [CrossRef]

23. Deng, Y.; Cheng, J.C.P.; Anumba, C. A framework for 3D traffic noise mapping using data from BIM and GIS integration. Struct. Infrastr. Eng. 2016, 12, 1267-1280. [CrossRef]

24. Nagel, C.; Stadler, A.; Kolbe, T.H. Conceptual Requirements for the Automatic Reconstruction of Building Information Models from Uninterpreted 3D Models. In Academic Track of Geoweb 2009: Cityscapes; ISPRS: Berlin, Germany, 2009; Volume XXXVIII-3-4/C3.

25. Cheng, J.C.; Deng, Y.; Anumba, C. Mapping BIM schema and 3D GIS schema semi-automatically utilizing linguistic and text mining techniques. J. Inf. Technol. Constr. 2015, 20, 193-212.

26. Schürr, A. Specification of graph translators with triple graph grammars. In Graph-Theoretic Concepts in Computer Science; Springer: Berlin/Heidelberg, Germany, 1995; pp. 151-163. [CrossRef]

27. Bardohl, R.; Ehrig, H.; de Lara, J.; Taentzer, G. Integrating Meta-modelling Aspects with Graph Transformation for Efficient Visual Language Definition and Model Manipulation. In Procceedings of the Fundamental Approaches to Software Engineering (FASE), Barcelona, Spain, 29 March-2 April 2004; Wermelinger, M., Margaria-Steffen, T., Eds.; Springer: Berlin/Heidelberg, Germany, 2004; pp. 214-228.

28. Westphal, M.; Rahm, T. Methoden zur automatischen Modelltransformation für die Simulation des maschinellen Tunnelvortriebs (Methods for the automatic model transformation for the simulation of mechanized tunneling operations). In Proceedings of the Forum Bauinformatik, Munich, Germany, 18-20 September 2013; pp. 135-146.

29. Rahm, T. Simulation-Based Evaluation of Disturbances of Production and Logistic Processes in Mechanized Tunneling Operations. Ph.D. Thesis, Ruhr-Universität Bochum, Bochum, Germany, March 2017.

30. Amelunxen, C.; Legros, E.; Schürr, A.; Stürmer, I. Checking and Enforcement of Modeling Guidelines with Graph Transformations. In Applications of Graph Transformations with Industrial Relevance; Schürr, A., Nagl, M., Zündorf, A., Eds.; Springer: Berlin/Heidelberg, Germany, 2008; pp. 313-328.

31. Jakob, J.; Schürr, A. View Creation of Meta Models by Using Modified Triple Graph Grammars. Electron. Notes Theor. Comput. Sci. 2008, 211, 181-190. [CrossRef]

32. Aschenbrenner, N.; Geiger, L. Transforming Scene Graphs Using Triple Graph Grammars: A Practice Report. In Applications of Graph Transformations with Industrial Relevance; Schürr, A., Nagl, M., Zündorf, A., Eds.; Springer: Berlin/Heidelberg, Germany, 2008; pp. 32-43.

33. Ehrig, H.; Ehrig, K. Overview of Formal Concepts for Model Transformations Based on Typed Attributed Graph Transformation. Electron. Notes Theor. Comput. Sci. 2006, 152, 3-22. [CrossRef] 
34. Ehrig, H.; Ehrig, K.; Prange, U.; Taentzer, G. Fundamentals of Algebraic Graph Transformation. In Monographs in Theoretical Computer Science. An EATCS Series; Springer: Berlin, Germany, 2006. [CrossRef]

35. Ehrig, H.; Ermel, C.; Golas, U.; Hermann, F., Model Transformation and Model Integration. In Graph and Model Transformation: General Framework and Applications; Springer: Berlin/Heidelberg, Germany, 2015; pp. 171-213.

36. Czarnecki, K.; Helsen, S. Feature-based Survey of Model Transformation Approaches. IBM Syst. J. Model-Driv. Softw. Dev. 2006, 45, 621-645. [CrossRef]

37. Taentzer, G.; Ehrig, K.; Guerra, E.; de Lara, J.; Lengyel, L.; Levendovszky, T.; Prange, U.; Varró, D.; Varro-Gyapay, S. Model Transformation by Graph Transformation: A Comparative Study. Available online: https: / / repositorio.uam.es/handle/10486/665862 (accessed on 20 May 2018).

38. Greenyer, J.; Kindler, E. Reconciling TGGs with QVT. In Proceedings of the 10th International Conference on Model Driven Engineering Languages and Systems (MODELS'07), Nashville, TN, USA, 30 September-5 October 2007; Springer: Berlin/Heidelberg, Germany, 2007; pp. 16-30.

39. Schürr, A.; Klar, F. 15 Years of Triple Graph Grammars: Research Challenges, New Contributions, Open Probelms. In Proceedings of the 4th International Conference on Graph Transformations (ICGT2008), Leicester, UK, 7-13 September 2008; Ehrig, H., Heckel, R., Rozenberg, G., Taentzer, G., Eds.; Springer: Berlin/Heidelberg, Germany, 2008; pp. 411-425.

40. ISO. Industrial Automation Systems and Integration. Product Data Representation and Exchange. Part 11: Description Methods: The EXPRESS Language Reference Manual; International Standard ISO 10303-11; International Organization For Standardization: Geneva, Switzerland, 2004.

41. ISO/IEC. Information Technology. Object Management Group Unified Modeling Language (OMG UML). Part 1: Infrastructure; International Standard ISO 19505-1; International Organization for Standardization: Geneva, Switzerland, 2012.

42. Tauscher, H.; Crawford, J. Graph representations for querying, examination, and analysis of IFC data. In Proceedings of the 12th European Conference on Product and Process Modelling (ECPPM), Copenhagen Denmark, 12-14 September 2018.

43. Lambers, L. A New Version of GTXL : An Exchange Format for Graph Transformation Systems. Electron. Notes Theor. Comput. Sci. 2005, 127, 51-63. [CrossRef]

44. Kindler, E.; Wagner, R. Triple Graph Grammars: Concepts, Extensions, Implementations, and Application Scenarios. In Proceedings of the MODELS: International Conference on Model Driven Engineering Languages and Systems, Nashville, TN, USA, 30 September-5 October 2007.

45. Konde, A.; Tauscher, H.; Biljecki, F.; Crawford, J. Floor plans in CityGML. ISPRS Ann. Photogramm. Remote Sens. Spat. Inf. Sci. 2018, in press.

46. Biljecki, F.; Kumar, K.; Nagel, C. CityGML Application Domain Extension (ADE): Overview of developments. Open Geosp. Data Softw. Stand. 2018, 3, 13. [CrossRef]

47. Open Geospatial Consortium. OGC City Geography Markup Language (CityGML) Encoding Standard 2.0.0; OGC Standard; Open Geospatial Consortium: Wayland, MA, USA, 2012.

48. Agugiaro, G.; Benner, J.; Cipriano, P.; Nouvel, R. The Energy Application Domain Extension for CityGML: Enhancing interoperability for urban energy simulations. Open Geosp. Data Softw. Stand. 2018, 3, 139. [CrossRef]

49. Biljecki, F.; Stoter, J.; Ledoux, H.; Zlatanova, S.; Çöltekin, A. Applications of 3D City Models: State of the Art Review. ISPRS Int. J. Geo-Inf. 2015, 4, 2842-2889. [CrossRef]

50. Southall, R.; Biljecki, F. The VI-Suite: A set of environmental analysis tools with geospatial data applications. Open Geosp. Data Softw. Stand. 2017, 2, 23. [CrossRef]

51. van den Brink, L.; Stoter, J.; Zlatanova, S. UML-Based Approach to Developing a CityGML Application Domain Extension. Trans. GIS 2013, 17, 920-942. [CrossRef]

52. Kumar, K.; Ledoux, H.; Commandeur, T.J.F.; Stoter, J.E. Modelling urban noise in CityGML ADE: Case of the Netherlands. ISPRS Ann. Photogramm. Remote Sens. Spat. Inf. Sci. 2017, IV-4-W5, 73-81. [CrossRef]

53. Löwner, M.O.; Gröger, G.; Benner, J.; Biljecki, F.; Nagel, C. Proposal for a new LOD and multi-representation concept for CityGML. ISPRS Ann. Photogramm. Remote Sens. Spat. Inf. Sci. 2016, IV-2/W1, 3-12.

54. Egusquiza, A.; Prieto, I.; Romero, A. Multiscale information management for sustainable districts rehabilitation EFFESUS and FASUDIR projects. In Proceedings of the 10th European Conference on Product and Process Modelling (ECPPM), Vienna, Austria, 17-19 September 2014; pp. 303-308. 
55. Prieto, I.; Izkara, J.L.; del Hoyo, F.J.D. Efficient Visualization of the Geometric Information of CityGML: Application for the Documentation of Built Heritage. In Computational Science and Its Applications_ICCSA 2012; Springer: Berlin/Heidelberg, Germany, 2012; pp. 529-544.

56. Karan, E.P.; Irizarry, J. Extending BIM interoperability to preconstruction operations using geospatial analyses and semantic web services. Autom. Constr. 2015, 53, 1-12. [CrossRef]

(C) 2018 by the authors. Licensee MDPI, Basel, Switzerland. This article is an open access article distributed under the terms and conditions of the Creative Commons Attribution (CC BY) license (http:// creativecommons.org/licenses/by/4.0/). 